\title{
e23 Unusual Presentation of An latrogenic Esophageal Perforation in A Newborn
}

Santosh Kumar Singh, Sunil Taneja and Rupa Dalmia Singh

Madhuraj Nursing Home, Kanpur, UP, India.

Abstract. Spontaneous rupture of esophagus (Boerhaave's Syndrome) in neonates is a rare occurrence. However iatrogenic perforation of the esophagus is not that uncommon, especially in a premature. The presentation of esophageal perforation is rather stereotyped. In the present case however patient presented with unusual features.

[Indian J Pediatr 2005; 72 (1) : e23-e25] E-mail: drsantosh6@rediffmail.com

\section{e26 Metastatic Gastric Adenocarcinoma}

R. Bhagwat, G. Biswas, A. Khadwal, S.K. Pai, S. Banavali and P. Parikh

Department of Medical Oncology, Tata Memorial Hospital, Parel, Mumbai, India.

Abstract. Gastrointestinal tumors represent less than $5 \%$ of all pediatric neoplasms. Within this subgroup carcinomas are rare, especially that of stomach. The authors present this rare entity with an equally rare presentation.

[Indian J Pediatr 2005; 72 (1) : e26-e28] E-mail: purvish@ rediffmail.com 